\section{SAT0532 POSITIVE DISEASE-SPECIFIC AUTOANTIBODIES LOWER DIAGNOSTIC SENSITIVITY BUT HAVE LITTLE CLINICAL SIGNIFICANCE IN DIAGNOSING IGG4- RELATED DISEASE USING THE 2019 ACR/EULAR CLASSIFICATION CRITERIA IN DAILY CLINICAL PRACTICE}

I. Mizushima ${ }^{1}$, T. Yamano ${ }^{1}$, H. Kawahara ${ }^{1}$, S. Hibino ${ }^{1}$, R. Nishioka ${ }^{1}$, T. Zoshima ${ }^{1}$, S. Hara ${ }^{1}$, K. Ito ${ }^{1}$, H. Fujii ${ }^{1}$, M. Kawano ${ }^{1}{ }^{1}{ }^{1}$ Kanazawa University Hospital, Rheumatology, Kanazawa, Japan

Background: Recently, the 2019 ACR/EULAR classification criteria for IgG4-related disease (IgG4-RD) were published mainly to identify more homogeneous subjects for inclusion in clinical trials and observational studies [1]. However although their high specificity is presumed to be useful to differentiate IgG4-RD from various mimickers, their value in daily clinical practice needs to be evaluated. Objectives: This study aimed to clarify the usefulness of the 2019 ACR/EULAR classification criteria for IgG4-RD and characteristics of false-negative patients in daily clinical practice.

Methods: We retrospectively reviewed the medical records of 162 patients with IgG4-RD and 130 consecutive non-IgG4-RD patients (mimickers) diagnosed by experts whose serum IgG4 levels were measured at a single center in Japan. Using the collected data, we calculated sensitivity, specificity, and fulfillment rates for the entry criteria, exclusion criteria, and threshold of inclusion criteria points. In addition, to clarify the characteristics of false-negative cases in IgG4-RD, we performed an intergroup comparison of their clinical features including disease-specific autoantibodies.

Results: Both the patients with IgG4-RD and mimickers were relatively old (66 and 65 years) with male predominance $(67 \%$ and $60 \%)$. The final diagnoses of mimickers mainly consisted of cancer, lymphoma, vasculitis, sarcoidosis, multicentric Castleman's disease, and atherosclerotic or infectious aortic aneurysm. The classification criteria had a sensitivity of $72.8 \%$ and a specificity of $100 \%$. Of the 44 false-negative cases, one did not fulfill the entry criteria, 20 fulfilled one exclusion criterion, and 27 did not achieve sufficient inclusion criteria points. Compared with the true-positive cases, the false-negative cases had significantly fewer affected organs, lower serum IgG4 levels, higher serum $\mathrm{CH} 50$ levels, and lower prevalence of salivary/lacrimal gland and renal parenchymal lesions. They were also less likely to have had biopsies ( $61 \%$ vs $97 \%$ ). Of note, positivity of disease-specific autoantibodies including SSA/Ro antibody, ANCA, ds-DNA antibody, and ACPA was the most common exclusion criterion fulfilled in 18 patients, only 2 of whom were diagnosed with a specific autoimmune disease (rheumatoid arthritis) complicated by IgG4-RD. The remaining 16 patients had no specific clinical symptoms related to such autoantibodies. In addition, compared with IgG4-RD patients without disease-specific autoantibodies, the 18 patients with them had almost equal serum IgG4 and complement levels, number of affected organs, and histopathology and immunostaining scores despite higher serum IgG and CRP levels.

Conclusion: The present study suggests that the 2019 ACR/EULAR classification criteria for IgG4-RD has excellent diagnostic specificity and moderate sensitivity in daily clinical practice. Positive disease-specific autoantibodies alone, which lowered the sensitivity in this study, may have little clinical significance concerning the diagnosis of IgG4-RD.

References:

[1] Wallace ZS et al. The 2019 American College of Rheumatology/European League Against Rheumatism classification criteria for lgG4-related disease. Ann Rheum Dis. 2020 Jan;79(1):77-87.

Disclosure of Interests: None declared

DOI: 10.1136/annrheumdis-2020-eular.3177

\section{SAT0533 ADULT ONSET STILLS DISEASE AND IDIOPATHIC RECURRENT PERICARDITIS: ARE THE MORE SIMILARITIES OR DIFFERENCES?}

V. Myachikova' ${ }^{1}$, O. Moiseeva' ${ }^{1}$, E. Kuvardin ${ }^{1}$, S. Lapin ${ }^{2}$, O. Tkachenko², V. Nazarov ${ }^{2}$, A. Maslyanskiy ${ }^{1}{ }^{1}$ Federal State Budgetary Institution "Almazov National Medical Research Centre" of the Ministry of Health of the Russian Federation, Sankt-Peterburg, Russian Federation; ${ }^{2}$ Pavlov First State Medical University of St. Petersburg, Sankt-Peterburg, Russian Federation

Background: Adult onset Stills disease (AOSD) and Idiopathic recurrent pericarditis (IRP) are currently considered auto-inflammatory diseases. Common features of these disorders are symptoms such as fever, leukocytosis, serositis, increased acute phase reactants.

Diagnosis of IRP is based on ESC 2015 diagnostic criteria, while AOSD is defined according to 3 sets of classification criteria. A detailed study shows that modern criteria for these nosologies overlap and do not allow distinguishing one from the other. Objectives: We have not found any data on the comparison of the two groups in the literature. We compared the two groups of patients according to several parameters, such as clinical features, laboratory testing, genetic analysis to identify common patterns.
Methods: We enrolled 22 newly identified subjects (13 patients with AOSD, 9 patients with IRP) to our prospective, monocenter study. The mean age of patients with AOSD was 31 [22; 39], the mean age of patients with IRP was 46 $[35 ; 54]$. Blood sampling in all patients was performed in the flare.

We quantified the serum levels of ferritin and its glycosylated fraction in both groups. Mutations of the MEFV, TNFRSF1A genes were studied. As more sensitive imaging methods for lymphadenopathy and serositis, we performed the following instrumental studies for all patients: transthoracic echocardiography, ultrasound of the abdominal cavity and pelvis, chest high-resolution computed tomography.

Results: One subject with a heterozygous missense variant was found in exon 2 of the MEFV gene (E148Q) in the IRP group. The patient was excluded from our study. Elevated white blood cell (WBC) count and C-reactive protein (CRP) were observed in all patients in 2 groups, however, the level of WBC greater than 10,000/ $\mathrm{mm}^{3}$ was found only in 10 patients from the AOSD group and 5-from the IRP. Elevated ferritin level in both groups was detected. The number of subjects with high level of ferritin in the AOSD group reached $12(n=13)$, in the IRP group $-7(n=8)$.

The ferritin level appeared to be more significant in the AOSD group compared to the IRP group $(1521 \mathrm{ng} / \mathrm{ml}$ vs $408 \mathrm{ng} / \mathrm{ml} \mathrm{p}=0.0159)$ Figure 1 . In turn, lower glycosylated ferritin was recorded in 9 patients with AOSD $(n=13)$, and 7 - with IRP $(n=8)$. We have demonstrated a more significant decrease of glycosylated ferritin level in patients with AOSD in comparison to patients with IRP, which amounted ( $11 \%$ vs $37 \% p=0.0286)$ reference value $(38.6 \%-84.7 \%)$. Figure 1 .

Abnormal liver function tests were found in the majority of patients with AOSD and IRP $(61 \%$ vs $75 \%)$

We have also shown that, if the patient had pericardial effusion, the fluid was present in the pleural cavity, regardless of the group.

The number of AOSD patients with polyserositis was $5(n=13)$.

Other symptoms are presented in Table 1

Table 1

\begin{tabular}{|c|c|c|}
\hline Symptom and sign & $\operatorname{AOSD}(n=13)$ & $\operatorname{IRP}(n=8)$ \\
\hline CRP mg/L, mean, \% & 123 [69;164], 100\% & $151[65 ; 226], 100 \%$ \\
\hline Pericarditis & $38 \%$ & $100 \%$ \\
\hline Pleuritis & $38 \%$ & $100 \%$ \\
\hline Leukocytosis $\geq 10,000 / \mathrm{mm} 3$ & $77 \%$ & $62 \%$ \\
\hline Abnormal liver function tests & $61 \%$ & $75 \%$ \\
\hline Fever $>39^{\circ} \mathrm{C}$ & $100 \%$ & $100 \%$ \\
\hline Rush & $77 \%$ & $0 \%$ \\
\hline Arthralgia & $100 \%$ & $75 \%$ \\
\hline Arthritis, lasting 2 weeks or longer & $100 \%$ & $12.5 \%$ \\
\hline Sore throat & $54 \%$ & $0 \%$ \\
\hline Recent Iymphadenopathy & $85 \%$ & $25 \%$ \\
\hline Hepatomegaly or splenomegaly & $54 \%$ & $62 \%$ \\
\hline Elevated ferritin & $92 \%$ & $87 \%$ \\
\hline Glycosylated ferritin $\leq 20 \%$ & $69 \%$ & $25 \%$ \\
\hline
\end{tabular}

Conclusion:

1. The level of ferritin in the IRP group was lower, which can be explained by a less generalized process, the absence of such symptoms as arthritis, rash, splenomegaly.

2. Diagnostic and classification criteria of both disorders do not allow distinguishing between the diseases.

3. There might be no differences between the diseases; further research (on more representative groups) is needed. We consider the comparison of the gene-expression analysis in these patients to be of great importance.

\section{Figure 1}
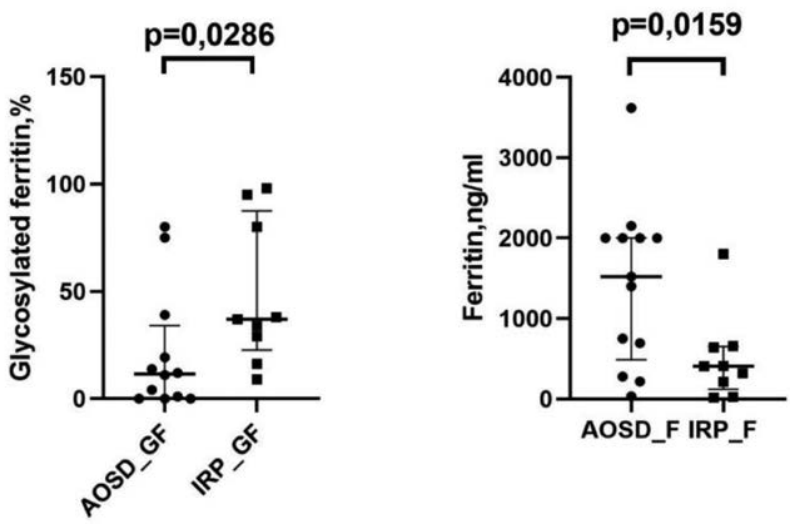

Disclosure of Interests: None declared DOI: 10.1136/annrheumdis-2020-eular.4916 